\title{
Diabetic Ketoacidosis With Acute Necrotizing Pancreatitis as First Presentation in Latent Autoimmune Diabetes in Adult
}

\author{
Stella Pak ${ }^{\mathrm{a}, \mathrm{d}}$, David Cha ${ }^{\mathrm{b}}$, Omeed Jazayeri-Moghaddas ${ }^{\mathrm{a}}$, Dexter Nye ${ }^{\mathrm{b}}$, Jillian Costello ${ }^{\mathrm{c}}$, \\ John-Phillip Markovic ${ }^{\text {a }}$ Jim Kim ${ }^{\mathrm{a}}$
}

\begin{abstract}
This case describes delayed diagnosis of acute necrotizing pancreatitis in the setting of diabetic ketoacidosis (DKA) as the first presentation of late onset autoimmune diabetes of adulthood (LADA). Possible presentation of DKA with LADA and acute pancreatitis (AP) are explored in this paper, with an emphasis on early diagnosis of pancreatitis for appropriate management. This case is a critical reminder that although DKA can non-specifically elevate pancreatic enzymes, LADA and AP can present concurrently, and pancreatic pathology should still be considered in the differential.
\end{abstract}

Keywords: Diabetic ketoacidosis; Acute pancreatitis; Latent autoimmune diabetes in adult

\section{Introduction}

Late onset autoimmune diabetes of adulthood (LADA) is a disorder characterized by the slow autoimmune destruction of pancreatic $\beta$-cells. The current diagnostic criteria for LADA include: 1) being over 30 years of age, 2) not requiring insulin for 6 months, 3) having specific diabetes associated autoantibodies, including autoantibodies to glutamic acid decarboxylase 65 (GADA), insulinoma-associated antigen IA-2 (IA-2A), islet cells (ICA) and zinc transporter 8 (ZnT8A) [1]. LADA is seen in $2-12 \%$ of all diabetic cases, but is presumed to be underdiagnosed due to the difficulty of making the diagnosis clinically [2]. In some unusual cases, the first presentation of LADA is diabetic ketoacidosis (DKA). Due to the slow progression of $\beta$-cell destruction in LADA, baseline insulin deficiency can be clinically undetected in some cases [3]. If an LADA patient is undiagnosed and encounters a stressful event,

Manuscript submitted September 16, 2017, accepted October 3, 2017

${ }^{a}$ Department of Internal Medicine, Kettering Medical Center, Kettering, OH, USA

${ }^{b}$ Wright State University School of Medicine, Dayton, OH, USA

'Liberty University College of Osteopathic Medicine, Lynchburg, VA, USA

${ }^{\mathrm{d}}$ Corresponding Author: Stella Pak, Department of Internal Medicine, Kettering Medical Center, 3535 Southern Blvd, Kettering, OH 45429, USA.

Email: stella.pak@rockets.utoledo.edu

doi: https://doi.org/10.14740/jmc2881w such as prolonged fasting or infection, insulin requirements are increased and DKA could be the outcome.

One other potential complication of DKA is coexisting acute pancreatitis (AP), which is seen in patients with or without LADA. AP as a complication of DKA is seen in $10-15 \%$ of cases and is a complex diagnosis to make. The complexity of this relationship stems from the clinical similarity between the presentations. DKA patients can have symptoms which mask AP, such as abdominal pain and elevated amylase and lipase ( $21 \%$ and $29 \%$ in patients, respectively) [4]. In DKA cases where AP is suspected, computed tomography (CT) can be invaluable in diagnosing AP. We report a case of a 32-year-old male diagnosed with DKA as the first presentation of LADA, which was complicated by AP.

\section{Case Report}

A 32-year-old male presented with altered mental status and history of increasing general weakness and vomiting in the past 3 days. Upon presentation, he was unresponsive. He had a blood pressure of $75 / 46 \mathrm{~mm} \mathrm{Hg}$, heart rate of 117 beats / $\mathrm{min}$, respiration rate of 24 breaths/min, oxygen saturation rate of $95 \%$ at room temperature, and body temperature of 37.1 ${ }^{\circ} \mathrm{C}$. He was noted to have blood glucose of $2,023 \mathrm{mg} / \mathrm{dL}$, beta hydroxybutyrate of $12.91 \mathrm{mmol} / \mathrm{L}$, lactate of $3.7 \mathrm{mmol} / \mathrm{L}$, and WBC of $12,200 / \mathrm{mm}^{3}$. His HbAlc value was $15.1 \mathrm{mmol} / \mathrm{mol}$, and his venous blood gas measurements revealed a $\mathrm{pH}$ of 7.24 and $\mathrm{HCO}_{3}$ of $16 \mathrm{mmol} / \mathrm{L}$. He was noted to have acute kidney injury with the following results: blood urea nitrogen (BUN) $43 \mathrm{mg} / \mathrm{dL}$, creatinine $3.16 \mathrm{mg} / \mathrm{dL}$, and estimated glomerular filtration rate (eGFR) $23 \mathrm{~mL} / \mathrm{min} / 1.73 \mathrm{~m}^{2}$.

The patient did not have prior history of diabetes mellitus. Yet, he was now being managed for DKA. He was aggressively hydrated and received IV insulin. He continued to be hemodynamically unstable and was started on epinephrine. The patient was morbidly obese and appeared to be dehydrated. The cardiology team was consulted due to a rise in troponin ( 0.05 $\mathrm{ng} / \mathrm{mL}$ ), and an echocardiogram they had ordered showed an ejection fraction of $40 \%$.

His altered mental status prompted a need for a CT scan of his brain. The results were negative for acute processes. The patient continued to be aggressively fluid resuscitated with epinephrine for pressor support. However, the patient continued to deteriorate, became agitated, tachypneic, and was subsequently intubated. 


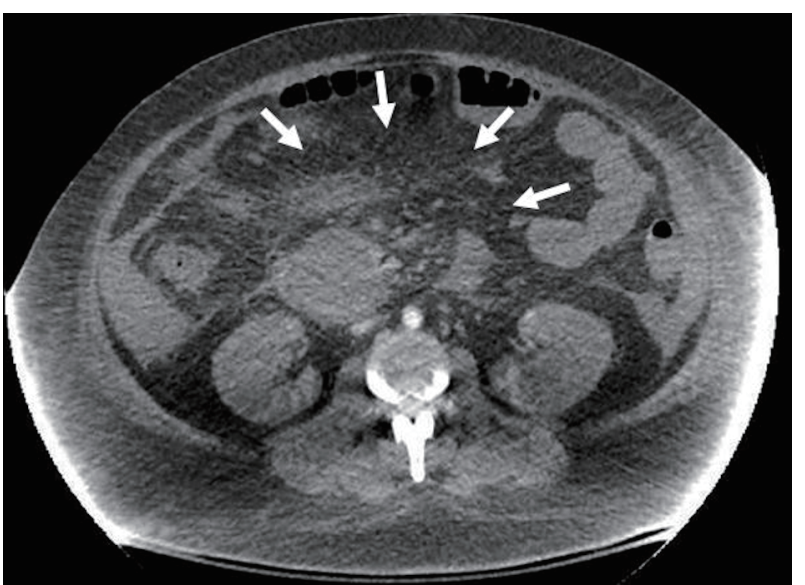

Figure 1. Abdomen computerized tomography without contrast showing severe necrotizing pancreatitis (arrows) with acute post-necrotic fluid accumulation and arterial hemorrhage from branches of the splenic artery.

Due to concern for intra-abdominal pathology, an abdomen $\mathrm{CT}$ without contrast was obtained showing evidence of pancreatitis. There was no evidence for gallstones. Blood work showed that his lipase and amylase were elevated at 890 and $391 \mathrm{U} / \mathrm{L}$, respectively. The patient persisted with metabolic acidosis as well as acute kidney injury. Subsequently, we started the patient on continuous veno-venous hemodialysis (CVVHD).

The patient was also noted to have disseminated intravascular coagulopathy (DIC) with a platelet count of 34,000/ $\mu \mathrm{L}$ necessitating expeditious plasmapheresis treatment. Additionally, a thrombus was found in the left internal jugular vein whereupon the patient was started on low intensity heparin drip.

Over the course of several days, the patient's hemoglobin was slowly decreasing from 17 to $6.8 \mathrm{~g} / \mathrm{dL}$. This was attributed to combination of plasmapheresis and CVVHD; nonetheless, a repeat CT of abdomen and pelvis showed a large fluid collection believed to be contributory to the progression of the pancreatitis.

Over 2 weeks, the patient appeared to recover uneventfully and was extubated. However, he suddenly developed acute abdominal pain as well as respiratory distress. The patient became unresponsive with hypertension (113/54 to $152 / 76 \mathrm{~mm}$ $\mathrm{Hg}$ ) with no palpable pulse. Cardiopulmonary resuscitation was performed and the patient received two doses of epinephrine with return of spontaneous circulation.

He was intubated again and restarted on epinephrine. He was found to have a significant drop in hemoglobin over $2 \mathrm{~h}$ ( 8.4 to $5.8 \mathrm{~g} / \mathrm{dL}$ ). His heparin drop was immediately discontinued. A CT of the abdomen with IV contrast demonstrated hemorrhagic pancreatitis (Fig. 1). Angioembolization of splenic artery failed due to severe tortuosity of the middle part of splenic artery and acute angle at which the splenic artery came off from the celiac artery.

After continued efforts with fluid, blood transfusions, pressors, and discussion with the family, he was taken to the operating room, undergoing exploratory laparotomy, evacuation of hemoperitoneum and lesser sac hematoma, and partial pancreatic necrosectomy to control the hemorrhage. On postoperative day 1, the patient's hemodynamic status improved with aggressive fluid resuscitation and use of approximately 20 units of blood products. Attempts at cannulation of the splenic artery for possible angioembolization were again made but unsuccessful. On postoperative day 2, the patient was taken back to the operating room for a second look laparotomy and evacuation of blood clots from the pancreatic bed and peri-splenic area. The patient was then intubated and sedated in critical condition.

Postoperatively, the patient continued to remain hemodynamically unstable and required more than 40 units of blood products and persistent acidosis despite massive transfusion, CVVHD, and multiple administration of epinephrine. The patient's poor prognosis was discussed extensively with the patient's family, ultimately electing for patient comfort care, and, on 16th day of hospitalization, the patient expired.

\section{Discussion}

The triad presentation of DKA, AP, and hypertriglyceridemia has been previously reported as a rare phenomenon that requires immediate diagnosis $[5,6]$. DKA may precipitate AP in patients due to increased lipolysis in the setting of insulin deficiency. Newly released free fatty acids cause an increased formation of very-low-density lipoproteins (VLDL) by the liver, resulting in hypertriglyceridemia. The increased triglyceride levels then induce a pro-inflammatory state, edema, and necrosis leading to pancreatitis $[7,8]$. In this case however, our patient's triglyceride level was only 249 U/L (severe hypertriglyceridemia $>1,000 \mathrm{U} / \mathrm{L}$ ), which leads us to believe the origin of AP is less likely DKA, but acute stress from AP most likely induced and exacerbated DKA in our patient [9].

Diagnosis of AP in the midst of DKA is challenging as $19-46 \%$ of cases present with abdominal pain [10]. It is reported that $24.7-79.0 \%$ of patients with DKA have non-specific elevation of amylase or lipase. For this reason, DKA is reported to mask a co-existing AP in $10-15 \%$ of cases [11]. Timely diagnosis of AP in DKA is critical as it would change the course of treatment. AP tends to require more aggressive hydration, longer nil per os (NPO) period, and stricter serum glucose control. Also, timely diagnosis would allow clinicians to monitor for complications from AP [10]. Therefore, in patients complaining of abdominal pain despite their improving state of DKA, further investigation with CT scan of abdomen to rule out AP is crucial.

The co-occurrence of acute necrotizing pancreatitis with LADA can present a complex clinical picture. If not detected early, complications from pancreatitis can exacerbate DKA, regardless of diabetic management. This case is a critical reminder that if a patient presents with DKA and has elevated pancreatic enzymes, a diagnosis with pancreatic origin should remain in the differential.

In conclusion, it would be judicious for physicians to order laboratory evaluations of lipase and amylase to rule out AP in the setting of DKA. Elevations of amylase and lipase values are atypical in the setting of DKA and thus are somewhat in- 
dicative of an acute pancreatic process. Since clinical presentation of DKA and AP share many similarities, these values may help differentiate between two very different disease processes which require aggressive acute treatment.

\section{References}

1. O’Neal KS, Johnson JL, Panak RL. Recognizing and appropriately treating latent autoimmune diabetes in adults. Diabetes Spectr. 2016;29(4):249-252.

2. Nambam B, Aggarwal S, Jain A. Latent autoimmune diabetes in adults: A distinct but heterogeneous clinical entity. World J Diabetes. 2010;1(4):111-115.

3. Guglielmi C, Palermo A, Pozzilli P. Latent autoimmune diabetes in the adults (LADA) in Asia: from pathogenesis and epidemiology to therapy. Diabetes Metab Res Rev. 2012;28(Suppl 2):40-46.

4. Nadhem O, Nakhla E, Smalligan RD. Diabetic ketoacidosis as first presentation of latent autoimmune diabetes in adult. Case Rep Med. 2015;2015:821397.

5. Nair S, Pitchumoni CS. Diabetic ketoacidosis, hyperlipidemia, and acute pancreatitis: the enigmatic triangle. Am
J Gastroenterol. 1997;92(9):1560-1561.

6. Wang Y, Attar BM, Bedrose S, et al. Diabetic ketoacidosis with hypertriglyceridemia-induced acute pancreatitis as first presentation of diabetes mellitus: report of three cases. AACE Clinical Case Reports. 2017;3(3).

7. Kimura W, Mossner J. Role of hypertriglyceridemia in the pathogenesis of experimental acute pancreatitis in rats. Int J Pancreatol. 1996;20(3):177-184.

8. Nair S, Yadav D, Pitchumoni CS. Association of diabetic ketoacidosis and acute pancreatitis: observations in 100 consecutive episodes of DKA. Am J Gastroenterol. 2000;95(10):2795-2800.

9. Kabadi UM. Pancreatic ketoacidosis: ketonemia associated with acute pancreatitis. Postgrad Med J. 1995;71(831):32-35.

10. Wang Y, Attar BM, Bedrose S, et al. Diabetic ketoacidosis with hypertriglyceridemia-induced acute pancreatitis as first presentation of diabetes mellitus: report of three cases. AACE Clinical Case Reports. 2016;3:e195-e199.

11. Hahn SJ, Park JH, Lee JH, Lee JK, Kim KA. Severe hypertriglyceridemia in diabetic ketoacidosis accompanied by acute pancreatitis: case report. J Korean Med Sci. 2010;25(9):1375-1378. 